\title{
The Number of Solutions of Cubic Equations over $\mathbb{Q}_{3}$ \\ (Beberapa Penyelesaian Persamaan Kubik ke atas $\mathbb{Q}_{3}$ )
}

\author{
MANSOOR SABUROV* \& MOHD Ali KHAMEINI AHMAD
}

ABSTRACT

We provide the number of solutions of a cubic equation in domains $\mathbb{Z}_{3}^{*}, \mathbb{Z}_{3} \backslash \mathbb{Z}_{3}^{*}, \mathbb{Q}_{3} \backslash \mathbb{Z}_{3}$ and $\mathbb{Q}_{3}$.

Keywords: Cubic equation; p-adic number; the number of solutions

ABSTRAK

Kami menyediakan beberapa penyelesaian persamaan kubik dalam domain $\mathbb{Z}_{3}^{*}, \mathbb{Z}_{3} \backslash \mathbb{Z}_{3}^{*}, \mathbb{Q}_{3} \backslash \mathbb{Z}_{3}$ dan $\mathbb{Q}_{3}$.

Kata kunci: Beberapa penyelesaian; nombor p-adic; persamaan kubik

\section{INTRODUCTION}

This study is a continuation of papers by Mukhamedov et al. (2014, 2013), Mukhamedov and Saburov (2013) and Saburov and Ahmad $(2015,2014)$ where a solvability criterion for a cubic equation over the $p$-adic field $\mathbb{Q}_{p}$ was provided. In this paper, we shall provide the number of solutions of cubic equation over domains $\mathbb{Z}_{3}^{*}, \mathbb{Z}_{3} \backslash \mathbb{Z}_{3}^{*}, \mathbb{Q}_{3} \backslash$ $\mathbb{Z}_{3}$ and $\mathbb{Q}_{3}$.

The field $\mathbb{Q}_{p}$ of $p$-adic numbers which was introduced by German mathematician K. Hensel was motivated primarily by an attempt to bring the ideas and techniques of the power series into number theory. Their canonical representation is analogous to the expansion of analytic functions into power series. This is one of the manifestations of the analogy between algebraic numbers and algebraic functions.

For a fixed prime $p$, by $\mathbb{Q}_{p}$ it is denoted the field of $p$-adic numbers, which is a completion of the rational numbers $\mathbb{Q}$ with respect to the non-Archimedean norm $|\cdot|_{p}: \mathbb{Q} \rightarrow \mathbb{R}$ given by:

$$
|x|_{p}= \begin{cases}p^{-r} & x \neq 0, \\ 0, & x=0,\end{cases}
$$

here, $x=p^{r} \frac{m}{n}$ with $r, m \in \mathbb{Z}, n \in \mathbb{N},(m, p)=(n, p)=1$. A number $r$ is called $a p$-order of $x$ and it is denoted by $\operatorname{ord}_{p}(x)=r$.

Any $p$-adic number $x \in \mathbb{Q}_{p}$ can be uniquely represented in the following canonical form

$$
x=p^{o r d}(x)\left(x_{0}+x_{1} \cdot p+x_{2} \cdot p_{2}+\ldots\right)
$$

where $x_{0} \in\{1,2, \ldots p-1\}$ and $x_{i} \in\{0,1,2, \ldots p-1\}, i \geq 1$, (Borevich \& Shafarevich 1966; Koblitz 1984).

More recently, numerous applications of $p$-adic numbers have shown up in theoretical physics and quantum mechanics (Beltrametti \& Cassinelli 1972; Khrennikov 1994, 1991; Volovich 1987).
Unlike the field $\mathbb{R}$ of real numbers, in general, the cubic equation $a x^{3}+b x^{2}+c x+d=0$ is not necessary to have a solution in $\mathbb{Q}_{p}$, where $a, b, c, d \in \mathbb{Q}_{p}$ with $a \neq 0$. For example, the following simple cubic equation $x^{3}=p$ does not have any solution in $\mathbb{Q}_{p}$. Therefore, it is natural to find a solvability criterion for the cubic equation in $\mathbb{Q}_{p}$. One of methods to find solutions of the cubic equation in a local field is the Cardano method. However, by means of the Cardano method, we could not tell an existence of solutions of any cubic equations (Mukhamedov et al. 2014, 2013).

To the best of our knowledge, we could not find the solvability criterion in an explicit form for the cubic equation in the Bible books of $p$-adic analysis and algebraic number theory (Apostol 1972; Cohen 2007; Gouvea 1997; Koblitz 1984; Lang 1994; Neukirch 1999, Schikhof 1984; Serre 1979). The solvability criterion for the cubic equation over $\mathbb{Q}_{p}$, for all prime $p$, was provided in papers by Mukhamedov et al. $(2014,2013)$ and Saburov and Ahmad $(2015,2014)$.

We know that, by means of suitable substitutions, any cubic equation can be written as a depressed cubic equation form

$$
x^{3}+a x=b,
$$

where $a, b \in \mathbb{Q}_{p}$. It is worth mentioning that there are some cubic equations which do not have any solutions in $\mathbb{Z}_{p}^{*}$ (resp. in $\mathbb{Z}_{p}$ ) but have solutions in $\mathbb{Z}_{p}$ (resp. in $\mathbb{Q}_{p}$ ) (Mukhamedov et al. 2014, 2013). Therefore, finding a solvability criterion for the depressed cubic equation (3) in domains $\mathbb{Z}_{3}^{*}, \mathbb{Z}_{3}, \mathbb{Z}_{3}$ $\backslash \mathbb{Z}_{3}^{*}, \mathbb{Q}_{3} \backslash \mathbb{Z}_{3}$ and $\mathbb{Q}_{3}$ is of independent interest. In Saburov and Ahmad (2015), the solvability criterion for a cubic equation in the domains $\mathbb{Z}_{3}^{*}, \mathbb{Z}_{3}$ and $\mathbb{Q}_{3}$ was presented. In this paper, without proof, we provide a solvability criterion for a cubic equation in the domains $\mathbb{Z}_{3} \backslash \mathbb{Z}_{3}^{*}, \mathbb{Q}_{3} \backslash \mathbb{Z}_{3}$ and $\mathbb{Q}_{3}$ as a complementary part of our previous research.

The solvability criterion and the number of solutions for the cubic equation (2) over the finite field $\mathbb{F}_{p}=\mathbb{Z} / p \mathbb{Z}$, 
where $a, b \in \mathbb{F}_{p}$, was provided in Serre (2003) and Sun (2007, 1998). Since $\mathbb{F}_{p}$ is a subgroup of $\mathbb{Q}_{p}$, our results extend the results in Serre (2003) and Sun $(2007,1998)$.

\section{PRELIMINARIES}

In this section, we shall present some necessary notations and notions from the $p$-adic analysis.

Let

$$
\mathbb{Z}_{p}=\left\{x \in \mathbb{Q}_{p}:|x|_{p} \leq 1\right\}, \mathbb{Z}_{p}^{*}=\left\{x \in \mathbb{Q}_{p}:|x|_{p}=1\right\}
$$

be sets of $p$-adic integers and unities, respectively.

We know that any $p$-adic unity $x \in \mathbb{Z}_{p}^{*}$ has a unique canonical representation

$$
x=x_{0}+x_{1} \cdot p+x_{2} \cdot p^{2}+\ldots
$$

where $x_{0} \in\{1,2, \ldots p-1\}$ and $x_{i} \in\{0,1,2, \ldots p-1\}$ for any $i \geq 1$. Moreover, any nonzero $p$-adic number $x \in \mathbb{Q}_{p}$ has a unique representation of the form $x=\frac{x^{*}}{x}$, where $x^{*} \in \mathbb{Z}_{p}^{*}$.

Let us introduce some notations which will be used throughout this paper.

Let $x \in \mathbb{Q}_{p}$ be a nonzero $p$-adic number and $x=\frac{x^{*}}{|x|_{p}}$ where $x^{*} \in \mathbb{Z}_{p}^{*}$ with

$$
x^{*}=x_{0}+x_{1} p+x_{2} p^{2}+\ldots+x_{k} p^{k}+\ldots
$$

where $x_{0} \in\{1, \ldots, p-1\}$ and $x_{i} \in\{0,1, \ldots, p-1\}$ for any $i \in \mathbb{N}$.

For given numbers $i_{0}, j_{0} \in\{1, \ldots, p-1\}$ and $i_{1}, \ldots, i_{k}$, $j_{1}, \ldots, j_{l} \in\{0,1, \ldots, p-1\}$, we define the following sets

$$
\begin{aligned}
& \mathbb{Z}_{p}^{*}\left[i_{0}, i_{1}, \ldots, i_{k}\right]=\left\{x^{*} \in \mathbb{Z}_{p}^{*}: x^{*}=i_{0}+i_{1} p+\ldots+\right. \\
& \left.i_{k} p^{k}+x_{k+1} p^{k+1}+\ldots\right\} \\
& \mathbb{Z}_{p}^{*}\left[i_{0}, i_{1}, \ldots, i_{k} \mid j_{0}, j_{1}, \ldots, j_{1}\right]=\mathbb{Z}_{p}^{*}\left[i_{0}, i_{1}, \ldots, i_{k}\right] \times \\
& \mathbb{Z}_{p}^{*}\left[j_{0}, j_{1}, \ldots, j_{1}\right]
\end{aligned}
$$

Let us consider the following sets in $\mathbb{Z}_{3}^{*} \times \mathbb{Z}_{3}^{*}$

$$
\begin{aligned}
& \Delta=\Delta_{1} \cup \Delta_{2}, \\
& \Delta_{1}=\Delta_{11} \cup \Delta_{12} \cup \Delta_{13}, \\
& \Delta_{2}=\Delta_{21} \cup \Delta_{22} \cup \Delta_{23},
\end{aligned}
$$

where

$$
\begin{aligned}
& \Delta_{11}=\bigcup_{\substack{i=0 \\
j=0}}^{2} \mathbb{Z}_{3}^{*}[2, i, j \mid 1,2, i, j], \\
& \Delta_{12}=\bigcup_{j=0}^{2} \mathbb{Z}_{3}^{*}[2,1, j \mid 1,2,1, j+1], \\
& \Delta_{13}=\bigcup_{\substack{i=1 \\
j=0}}^{2} \mathbb{Z}_{3}^{*}[2, i+1, j+1 \mid 1,2, i+1, j],
\end{aligned}
$$

$$
\begin{aligned}
& \Delta_{21}=\bigcup_{\substack{i=0 \\
j=0}}^{2} \mathbb{Z}_{3}^{*}[2, i+j, i \mid 2,0,2-(i+j), j], \\
& \Delta_{22}=\bigcup_{j=0}^{2} \mathbb{Z}_{3}^{*}[2,0,2-j \mid 2,0,2, j], \\
& \Delta_{23}=\bigcup_{\substack{i=1 \\
j=0}}^{2} \mathbb{Z}_{3}^{*}[2,3-i, j \mid 2,0, i-1,1-(i+j)],
\end{aligned}
$$

where all entries of $\mathbb{Z}_{3}^{*}[2, \ldots \mid 1, \ldots]$ and $\mathbb{Z}_{3}^{*}[2, \ldots \mid 2, \ldots]$ belong to the set $\{0,1,2$,$\} .$

Finally, Hensel's lemma would be a powerful tool in order to obtain the solvability criterion for the cubic equation in the domain $\mathbb{Z}_{3}$.

Lemma 3 (Hensel's Lemma, (Borevich \& Shafarevich 1966)) Let $f(x)$ be polynomial whose the coefficients are $p$-adic integers. Let $\theta$ be a $p$-adic integer such that for some $i \geq 0$ we have

$$
\begin{aligned}
& f(\theta) \equiv 0\left(\bmod p^{2 i+1}\right), \\
& f^{\prime}(\theta) \equiv 0\left(\bmod p^{i}\right), f^{\prime}(\theta) \not 0\left(\bmod p^{i+1}\right) .
\end{aligned}
$$

Then $f(x)$ has a unique $p$-adic integer root $x_{0}$ which satisfies $x_{0} \equiv \theta\left(\bmod p^{i+1}\right)$.

\section{SOLVABILITY CRITERIA OVER DOMAINS $\mathbb{Z}_{3} \backslash \mathbb{Z}_{3}^{*}$, AND $\mathbb{Q}_{3} \backslash \mathbb{Z}$}

In this section, we provide the solvability criterion of the cubic equation

$$
x^{3}+a x=b,
$$

over the domains $\mathbb{Z}_{3} \backslash \mathbb{Z}_{3}^{*}$ and $\mathbb{Q}_{3} \backslash \mathbb{Z}_{3}$ as a complementary part of Saburov and Ahmad (2015) paper, where $a, b \in$ $\mathbb{Q}_{3}$ and $a b \neq 0$. The proof is the similar to the proof in the case $\mathbb{Z}_{3}$ presented in Saburov and Ahmad (2015). However, for reasons of self-exposition, we present the solvability criterion in the domains $\mathbb{Z}_{3}^{*}, \mathbb{Z}_{3} \backslash \mathbb{Z}_{3}^{*}, \mathbb{Q}_{3} \backslash \mathbb{Z}_{3}$ and $\mathbb{Q}_{3}$. In the next section, we provide the number of solutions of cubic equations over the mentioned domains.

Theorem 4 Let $\Delta$ be the set given by (3). Then the following statements hold true:

I. The cubic equation (4) is solvable in $\mathbb{Z}_{3}^{*}$ if and only if either one of the following conditions holds true:

1. $|b|_{3}=|a|_{3}>1$;

2. $|b|_{3}=|a|_{3}=1 ; a^{*} \in \mathbb{Z}_{3}^{*}[1]$

3. $|b|_{3}<|a|_{3}=1 ; a^{*} \in \mathbb{Z}_{3}^{*}[2]$

4. $|a|_{3}<|b|_{3}=1$ and

$$
\begin{aligned}
& \text { i. }|a|_{3}=\frac{1}{3},\left(a^{*}, b^{*}\right) \in \mathbb{Z}_{3}^{*}[1 \mid 1,1] \cup \mathbb{Z}_{3}^{*}[1 \mid 2,1] \cup \Delta \text {; } \\
& \text { ii. }|a|_{3}<\frac{1}{3}, b^{*} \in \mathbb{Z}_{3}^{*}[1,0] \cup \mathbb{Z}_{3}^{*}[2,2] .
\end{aligned}
$$


II. The cubic equation (1) is solvable in $\mathbb{Z}_{3} \backslash \mathbb{Z}_{3}^{*}$ if and only if either one of the following conditions holds true:

1. $|a|_{3}^{3}>|b|_{3}^{2},|a|_{3}>|b|_{3}$

2. $|a|_{3}^{3}=|b|_{3}^{2}<1, \quad a^{*} \in \mathbb{Z}_{3}^{*}[1]$;

3. $|a|_{3}^{3}<|b|_{3}^{2}<1,\left.3\left|\log _{3}\right| b\right|_{3}$, and

i. $\quad\left|\frac{a}{3}\right|_{3}^{3}=|b|_{3}^{2},\left(a^{*}, b^{*}\right) \in \mathbb{Z}_{3}^{*}[1 \mid 1,1] \cup \mathbb{Z}_{3}^{*}[1 \mid 2,1] \cup \Delta ;$

ii. $\left|\frac{a}{3}\right|_{3}^{3}<|b|_{3}^{2}, b^{*} \in \mathbb{Z}_{3}^{*}[1,0] \cup \mathbb{Z}_{3}^{*}[2,2]$.

III. The cubic equation (1) is solvable in $\mathbb{Q}_{3} \backslash \mathbb{Z}_{3}$ iff either one of the following conditions holds true:

1. $|a|_{3}^{3}>|b|_{3}^{2}$ and
i. $|a|_{3}<|b|_{3}$;
ii. $|a|_{3} \geq|b|_{3},|a|_{3}>1,\left.2\left|\log _{3}\right| a\right|_{3}, a^{*} \in \mathbb{Z}_{3}^{*}[2]$;

2. $|a|_{3}^{3}=|b|_{3}^{2}>1, a^{*} \in \mathbb{Z}_{3}^{*}[1]$;

3. $|a|_{3}^{3}<|b|_{3}^{2},|b|_{3}>1,\left.3\left|\log _{3}\right| b\right|_{3}$, and

i. $\left|\frac{a}{3}\right|_{3}^{3}=|b|_{3}^{2},\left(a^{*}, b^{*}\right) \in \mathbb{Z}_{3}^{*}[1 \mid 1,1] \cup \mathbb{Z}_{3}^{*}[1 \mid 2,1] \cup \Delta$;

ii. $\left|\frac{a}{3}\right|_{3}^{3}<|b|_{3}^{2}, b^{*} \in \mathbb{Z}_{3}^{*}[1,0] \cup \mathbb{Z}_{3}^{*}[2,2]$.

IV. The cubic equation (1) is solvable in $\mathbb{Q}_{3}$ iff either one of the following conditions holds true:

1. $|a|_{3}^{3}>|b|_{3}^{2}$

2. $|a|_{3}^{3}=|b|_{3}^{2}, a^{*} \in \mathbb{Z}_{3}^{*}[1]$;

3. $|a|_{3}^{3}<|b|_{3}^{2},\left.3\left|\log _{3}\right| b\right|_{3}$, and

i. $\quad\left|\frac{a}{3}\right|_{3}^{3}=|b|_{3}^{2},\left(a^{*}, b^{*}\right) \in \mathbb{Z}_{3}^{*}[1 \mid 1,1] \cup \mathbb{Z}_{3}^{*}[1 \mid 2,1] \cup \Delta ;$

ii. $\left|\frac{a}{3}\right|_{3}^{3}<|b|_{3}^{2}, b^{*} \in \mathbb{Z}_{3}^{*}[1,0] \cup \mathbb{Z}_{3}^{*}[2,2]$.

\section{THE NUMBER OF SOLUTIONS OVER DOMAINS $\mathbb{Z}_{3}^{*}, \mathbb{Z}_{3} \backslash \mathbb{Z}_{3}^{*}, \mathbb{Q}_{3} \backslash \mathbb{Z}_{3}$ AND $\mathbb{Q}_{3}$}

In the next section, we provide the number of solutions of cubic equations over the domains $\mathbb{Z}_{3}^{*}, \mathbb{Z}_{3} \backslash \mathbb{Z}_{3}^{*}, \mathbb{Q}_{3} \backslash \mathbb{Z}_{3}$ and $\mathbb{Q}_{3}$.

Theorem 5 Let $\Delta$ be the set given by (3). Then the following statements hold true:

$N_{\mathbb{Z}_{3}^{*}}\left(x^{3}+a x-b\right)=\left\{\begin{array}{l}1,|b|_{3}=|a|_{3}>1 \\ 1,|a|_{3}=|b|_{3}=1, a^{*} \in \mathbb{Z}_{3}^{*}[1] \\ 2,|b|_{3}<|a|_{3}=1, a^{*} \in \mathbb{Z}_{3}^{*}[2] \\ 1,|a|_{3}<|b|_{3}=1,|a|_{3}=\frac{1}{3}, \\ \quad\left(a^{*}, b^{*}\right) \in \mathbb{Z}_{3}^{*}[1 \mid 2,1] \cup \mathbb{Z}_{3}^{*}[1 \mid 1,1] \cup \Delta \\ 1,|a|_{3}<|b|_{3}=1,|a|_{3}<\frac{1}{3}, b^{3} \in \mathbb{Z}_{3}^{*}[1,0] \cup \mathbb{Z}_{3}^{*}[2,2]\end{array}\right.$

$$
N_{\mathbb{Z}_{3} \backslash \mathbb{Z}_{3}^{*}}\left(x^{3}+a x-b\right)=\left\{\begin{array}{l}
3,|b|_{3}^{2}<|a|_{3}^{3}<1,2 \mid \underset{3}{\log |a|_{3}}, a^{*} \in \mathbb{Z}_{3}^{*}[2] \\
1,|b|_{3}^{2}<|a|_{3}^{3}<1,\left.2\left|\log _{3}\right| a\right|_{3}, a^{*} \notin \mathbb{Z}_{3}^{*}[2] \\
1,|b|_{3}^{2}<|a|_{3}^{3}<1,2 \underset{3}{\log |a|_{3}} \\
1,|b|_{3}^{2}<|a|_{3}^{3},|b|_{3}<|a|_{3},|a|_{3} \geq 1 \\
1,|a|_{3}^{3}=|b|_{3}^{2}<1, a^{*} \in \mathbb{Z}_{3}^{*}[1] \\
1,|a|_{3}^{3}<|b|_{3}^{2}<1,\left.3\left|\log _{3}\right| b\right|_{3},\left|\frac{a}{3}\right|_{3}^{3}=|b|_{3}^{2}, \\
\quad\left(a^{*}, b^{*}\right) \in \mathbb{Z}_{3}^{*}[1 \mid 2,1] \cup \mathbb{Z}_{3}^{*}[1 \mid 1.1] \cup \Delta \\
1,|a|_{3}^{3}<|b|_{3}^{2}<1,\left.3\left|\log _{3}\right| b\right|_{3},\left|\frac{a}{3}\right|_{3}^{3}<|b|_{3}^{2}, \\
b^{*} \in \mathbb{Z}_{3}^{*}[1,0] \cup \mathbb{Z}_{3}^{*}[2,2]
\end{array}\right.
$$

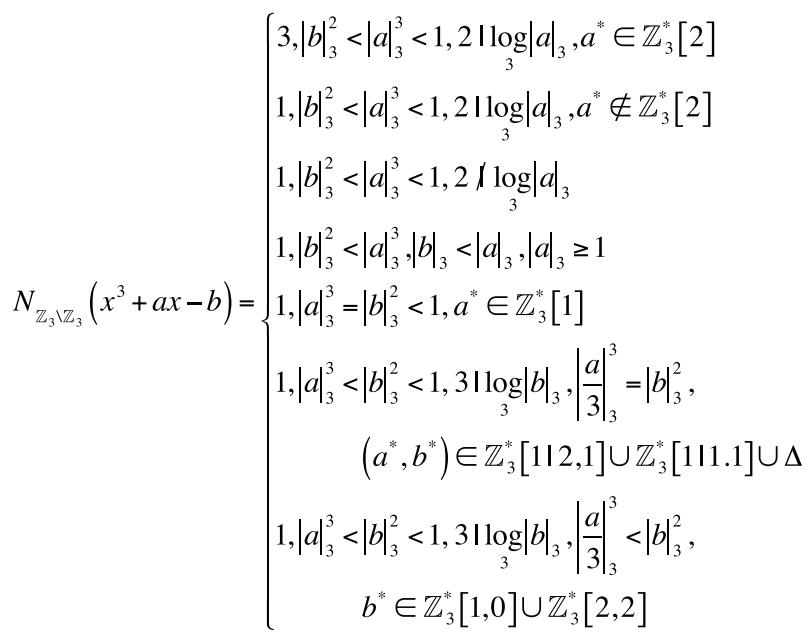

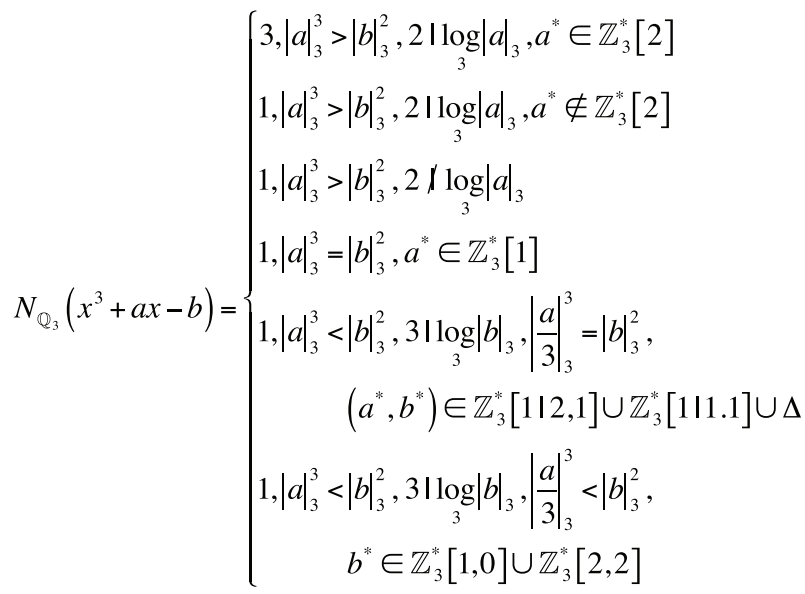

Proof The domain $\mathbb{Z}_{3}^{*}$ : the cubic equation (4) is solvable in $\mathbb{Z}_{3}^{*}$ if and only if either one of four conditions of Theorem 4 holds true. We want to find the number of solutions in every case. 
Case I.1: $|a|_{3}=|b|_{3}>1$. In this case, we showed (Saburov \& Ahmad 2015) that the number of solutions of the equation (4) in $\mathbb{Z}_{3}^{*}$ is the same as the number of solutions of the linear equation $a_{0} x_{0}=b_{0}$ in $\mathbb{F}_{3}$. Since $a_{0} \neq 0$, the last equation has a unique solution. Therefore, if $|a|_{p}=|b|_{p}>1$ then the depressed cubic equation (4) has a unique solution in $\mathbb{Z}_{3}^{*}$.

Case I.2: $|a|_{3}=|b|_{3}=1$ and $a^{*} \in \mathbb{Z}_{3}^{*}[1]$. In this case, we showed (Saburov \& Ahmad 2015) that the number of solutions of the equation (4) in $\mathbb{Z}_{3}^{*}$ is the same as the number of solutions of the equation $x_{0}^{3}+\mathrm{a}_{0} x_{0}=x_{0}+a_{0} x_{0}=b_{0}$ in $\mathbb{F}_{3}$. Since $a_{0}=1$, we have that $x_{0}=2 b_{0}$, a unique solution. Thus, if $|a|_{3}=|b|_{3}=1$ and $a^{*} \in \mathbb{Z}_{3}^{*}[1]$ then the cubic equation (4) has a unique solution in $\mathbb{Z}_{3}^{*}$.

Case I.3: $|b|_{3}<|a|_{3}=1$ and $a^{*} \in \mathbb{Z}_{3}^{*}[2]$. In this case, we showed (Saburov \& Ahmad 2015) that the number of solutions of the equation (4) in $\mathbb{Z}_{3}^{*}$ is the same as the number of solutions of the equation $x_{0}^{2}=-a_{0}$ in $\mathbb{F}_{3}$. Since $a_{0}=2$ the last equation has 2 distinct solutions in $\mathbb{F}_{3}$. Therefore, if $|b|_{3}<|a|_{3}=1$ and $a^{*} \in \mathbb{Z}_{3}^{*}[2]$ then the cubic equation (4) has 2 distinct solutions in $\mathbb{Z}_{3}^{*}$.

Case I.4. (i-a): $|a|_{3}<|b|_{3}=1,|a|_{3}=\frac{1}{3}$ and $\left(a^{*}, b^{*}\right) \in \mathbb{Z}_{3}^{*}[1 \mid 1,1]$ $\cup \mathbb{Z}_{3}^{*}[1 \mid 2,1]$. For this case, we showed (Saburov \& Ahmad 2015 ) that the number of solutions of the equation (4) in $\mathbb{Z}_{3}^{*}$ is the same as the number of solutions of the equation $x^{3}+a x-b \equiv 0(\bmod 27)$. Since $\left(a^{*}, b^{*}\right) \in \mathbb{Z}_{3}^{*}[1 \mid 1,1] \cup \mathbb{Z}_{3}^{*}$ $[1 \mid 2,1]$, then we have that $x \equiv b_{0}+3\left(b_{0}-1+a_{1} b_{0}-b_{2}\right)$ $(\bmod 9)$. Hence, if $|a|_{3}<|b|_{3}=1,|a|_{3}=\frac{1}{3}$ and $\left(a^{*}, b^{*}\right) \in \mathbb{Z}_{3}^{*}$ $[1 \mid 1,1] \cup \mathbb{Z}_{3}^{*}[1 \mid 2,1]$ then the cubic equation (4) has a unique solution in $\mathbb{Z}_{3}^{*}$.

Case I.4. (i-b): $|a|_{3}<|b|_{3}=1,|a|_{3}=\frac{1}{3}$ and $\left(a^{*}, b^{*}\right) \in \Delta$. For this case, we showed (Saburov \& Ahmad 2015) that the number of solutions of the equation (4) in $\mathbb{Z}_{3}^{*}$ is the same as the number of solutions of the equation $x^{3}+a x-b \equiv 0(\bmod$ 243). Since $\left(a^{*}, b^{*}\right) \in \Delta$, then we have a unique solution $x \equiv b_{0}+3 \bar{x}_{1}+9 \bar{x}_{2}(\bmod 27)$ where $\bar{x}_{1} \neq \equiv 1+a_{1} b_{0}(\bmod 3)$ (Saburov \& Ahmad 2015). Therefore, if $|a|_{3}<|b|_{3}=1,|a|_{3}$ $=\frac{1}{3}$ and $\left(a^{*}, b^{*}\right) \in \Delta$ then the depressed cubic equation (4) has a unique solution in $\mathbb{Z}_{3}^{*}$.

Case I.4 (ii): $|a|_{3}<|b|_{3}=1,|a|_{3}<\frac{1}{3}$ and $b^{*} \in \mathbb{Z}_{3}^{*}[1,0] \cup \mathbb{Z}_{3}^{*}$ $[2,2]$. In this case, we showed (Saburov \& Ahmad 2015) that the number of solutions of the equation (4) in $\mathbb{Z}_{3}^{*}$ is the same as the number of solutions of the equation $x^{3}+$ $a x-b \equiv 0(\bmod 27)$. Since $b^{*} \in \mathbb{Z}_{3}^{*}[1,0] \cup \mathbb{Z}_{3}^{*}[2,2]$, then we have that $x \equiv b_{0}+3\left(b_{2}-b_{0} a_{0}\right)(\bmod 9)$. So, if $\left.\left|a_{3}<\right| b\right|_{3}$ $=1,|a|_{3}<\frac{1}{3}$ and $b^{*} \in \mathbb{Z}_{3}^{*}[1,0] \cup \mathbb{Z}_{3}^{*}[2,2]$ then the depressed cubic equation (4) has a unique solution in $\mathbb{Z}_{3}^{*}$.

The domain $\mathbb{Z}_{3} \backslash \mathbb{Z}_{3}^{*}$ : the cubic equation (4) is solvable in $\mathbb{Z}_{3} \backslash \mathbb{Z}_{3}^{*}$ if and only if either one of three conditions of Theorem 4 holds true. We want to find the number of solutions in every case.
Case II.1: $|b|_{3}^{2}<|a|_{3}^{3}$ and $|b|_{3}<|a|_{3}$. Let us define the following sets

$$
\begin{aligned}
& \nabla=\left\{(a, b) \in \mathbb{Q}_{3}^{2}:|b|_{3}^{2}<|a|_{3}^{3},|b|_{3}<|a|_{3}\right\}, \\
& \nabla_{1}=\left\{(a, b) \in \mathbb{Q}_{3}^{2}:|b|_{3}^{2}<|a|_{3}^{3}<1,2 \mid \underset{3}{\log |a|_{3}}, a^{*} \in \mathbb{Z}_{3}^{*}[2]\right\}, \\
& \nabla_{2}=\left\{(a, b) \in \mathbb{Q}_{3}^{2}:|b|_{3}^{2}<|a|_{3}^{3}<1,2 \mid \underset{3}{\log |a|_{3}}, a^{*} \notin \mathbb{Z}_{3}^{*}[2]\right\}, \\
& \nabla_{3}=\left\{(a, b) \in \mathbb{Q}_{3}^{2}:|b|_{3}^{2}<|a|_{3}^{3}<1,2 \mid \underset{3}{\left.\log |a|_{3}\right\},}\right. \\
& \nabla_{4}=\left\{(a, b) \in \mathbb{Q}_{3}^{2}:|b|_{3}^{2}<|a|_{3}^{3},|b|_{3}^{3}<|a|_{3},|a|_{3} \geq 1\right\} .
\end{aligned}
$$

One can easily check that

$$
\nabla=\nabla_{1} \cup \nabla_{2} \cup \nabla_{3} \cup \nabla_{4}
$$

In the domain $\nabla_{1}$, we showed that the number of solutions of the depressed cubic equation (4) in the domain $\mathbb{Z}_{3} \backslash \mathbb{Z}_{3}^{*}$ is the same as the total number of solutions of the following two equations in the domain $\mathbb{Z}_{3}^{*}$ :

$$
\begin{aligned}
& y^{3}+a^{*} y=b \sqrt{|a|_{3}^{3}}, \\
& z^{3}+a\left|\frac{b}{a}\right|_{3}^{2} \quad z=b\left|\frac{b}{a}\right|_{3}^{3},
\end{aligned}
$$

It is clear that $\left|b \sqrt{|a|_{3}^{3}}\right|_{3}<\left|a^{*}\right|_{3}=1, a^{*} \in \mathbb{Z}_{3}^{*}[2]$ and $\left.\left.|a| \frac{b}{a}\right|_{3} ^{2}\right|_{3}=\left.\left.|b| \frac{b}{a}\right|_{3} ^{3}\right|_{3}>1$.

In this case, as we already proved, the equation (5) has 2 distinct solutions in $\mathbb{Z}_{3}^{*}$ and the equation (6) has a unique solution in $\mathbb{Z}_{3}^{*}$. Consequently, the depressed cubic equation (4) has 3 solutions in $\mathbb{Z}_{3} \backslash \mathbb{Z}_{3}^{*}$.

In the case $\nabla_{2} \cup \nabla_{3} \cup \nabla_{4}$, we showed that the number of solutions of the depressed cubic equation (4) in the domain $\mathbb{Z}_{3} \backslash \mathbb{Z}_{3}^{*}$ is the same as the number of solutions of the equation (6) in the domain $\mathbb{Z}_{3}^{*}$. We know that the equation (6) has a unique solution in $\mathbb{Z}_{3}^{*}$. Consequently, the depressed cubic equation (4) has a unique solution in $\mathbb{Z}_{3} \backslash \mathbb{Z}_{3}^{*}$.

Case II.2: $|a|_{3}^{3}=|b|_{3}^{2}<1$ and $a^{*} \in \mathbb{Z}_{3}^{*}[1]$. In this case, we showed that the number of solutions of the cubic equation (4) in the domain $\mathbb{Z}_{3} \backslash \mathbb{Z}_{3}^{*}$ is the same as the number of solutions of the following equation in the domain $\mathbb{Z}_{3}^{*}$

$$
y^{3}+a^{*} y=b^{*}
$$

It is clear that $\left|a^{*}\right|_{3}=\left|b^{*}\right|_{3}=1$ and $a^{*} \in \mathbb{Z}_{3}^{*}[1]$. We can apply Theorem 4 in order to get the number of solutions of the cubic equation (4). Consequently, if $|a|_{3}^{3}=|b|_{3}^{2}<1$ and $a^{*}$ $\in \mathbb{Z}_{3}^{*}[1]$ then the depressed cubic equation (7) has a unique solution in $\mathbb{Z}_{3} \backslash \mathbb{Z}_{3}^{*}$. 
Case II.3. (i): $|a|_{3}^{3}<|b|_{3}^{2}<1,\left.3\left|\log _{3}\right| b\right|_{3},\left|\frac{a}{3}\right|_{3}^{3}=|b|_{3}^{2},\left(a^{*}, b^{*}\right)$ $\in \mathbb{Z}_{3}^{*}[1 \mid 1,1] \cup \mathbb{Z}_{3}^{*}[1 \mid 2,1] \cup \Delta$. In this case, we showed that the number of solutions of the equation (4) in the domain $\mathbb{Z}_{3} \backslash \mathbb{Z}_{3}^{*}$ is the same as the number of solutions of the following equation in the domain $\mathbb{Z}_{3}^{*}$ :

$$
y^{3}+a^{3} \sqrt{|b|_{3}^{2}} y=b^{*}
$$

Then it is clear that $\frac{1}{3}=\left|a^{3} \sqrt{|b|_{3}^{2}}\right|_{3}<\left|b^{*}\right|_{3}=1$ and $\left(a^{*}, b^{*}\right)$ $\in \mathbb{Z}_{3}^{*}[1 \mid 1,1] \cup \mathbb{Z}_{3}^{*}[1 \mid 2,1] \cup \Delta$.

Consequently, if $|a|_{3}^{3}<|b|_{3}^{2}<1,\left.3\left|\log _{3}\right| b\right|_{3},\left|\frac{a}{3}\right|_{3}^{3}=|b|_{3}^{2},\left(a^{*}, b^{*}\right)$ $\in \mathbb{Z}_{3}^{*}[1 \mid 1,1] \cup \mathbb{Z}_{3}^{*}[1 \mid 2,1] \cup \Delta$ then the depressed cubic equation (4) has a unique solution in $\mathbb{Z}_{3} \backslash \mathbb{Z}_{3}^{*}$.

Case II.3. (ii): $|a|_{3}^{3}<|b|_{3}^{2}<1,\left.3\left|\log _{3}\right| b\right|_{3},\left|\frac{a}{3}\right|_{3}^{3}<|b|_{3}^{2}, b^{*} \in \mathbb{Z}_{3}^{*}$ $[1,0] \cup \mathbb{Z}_{3}^{*}[2.2]$. In this case, we showed that the number of solutions of the equation (4) in the domain $\mathbb{Z}_{3} \backslash \mathbb{Z}_{3}^{*}$ is the same as the number of solutions of the equation (8) in the domain $\mathbb{Z}_{3}^{*}$.

Then it is clear that $\left|a^{3} \sqrt{|b|_{3}^{2}}\right|_{3}<\frac{1}{3}<\left|b^{*}\right|_{3}=1$ and $b^{*} \in \mathbb{Z}_{3}^{*}$ $[1,0] \cup \mathbb{Z}_{3}^{*}[2,2]$. Consequently, if $|a|_{3}^{3}<|b|_{3}^{2}<1,\left.3\left|\log _{3}\right| b\right|_{3}$, $\left|\frac{a}{3}\right|_{3}^{3}<|b|_{3}^{2}$ and $b^{*} \in \mathbb{Z}_{3}^{*}[1,0] \cup \mathbb{Z}_{3}^{*}[2.2]$ then the depressed cubic equation (4) has a unique solution in $\mathbb{Z}_{3} \backslash \mathbb{Z}_{3}^{*}$.

The proofs for cases in the domains $\mathbb{Q}_{3} \backslash \mathbb{Z}_{3}$ and $\mathbb{Q}_{3}$ are similar to the case in the domain $\mathbb{Z}_{3} \backslash \mathbb{Z}_{3}^{*}$. This completes the proof.

\section{ACKNOWLEDGMENTS}

This work has been supported by ERGS13-025-0058. The first Author (M.S.) is also grateful to the Junior Associate scheme of the Abdus Salam International Centre for Theoretical Physics, Trieste, Italy.

\section{REFERENCES}

Apostol, T.M. 1972. Introduction to Analytic Number Theory. New York: Springer-Verlag.

Beltrametti, E. \& Cassinelli, G. 1972. Quantum mechanics and p-adic numbers Found. Phys. 2: 1-7.

Borevich, Z.I. \& Shafarevich, I.R. 1966. Number Theory. New York: Acad. Press.
Cohen, H. 2007. Number Theory, Volume I, II. New York: Springer.

Gouvea, F.Q. 1997. p-adic Numbers: An Introduction. Berlin: Springer-Verlag.

Khrennikov, A.Y. 1991. $p$-adic quantum mechanics with $p$-adic valued functions. J. Math. Phys. 32: 932.

Khrennikov, A.Y. 1994. p-Adic Valued Distributions in Mathematical Physics. Netherlands: Springer.

Koblitz, N. 1984. p-adic numbers, $p$-adic Analysis, and Zeta Functions, 2nd ed. New York: Springer.

Lang, S. 1994. Algebraic Number Theory. New York: SpringerVerlag.

Mukhamedov, F., Omirov, B. \& Saburov, M. 2014. On cubic equations over $p$-adic field. International Journal of Number Theory 10(5): 1171-1190.

Mukhamedov, F., Omirov, B., Saburov, M. \& Masutova, K. 2013. Solvability of cubic equations in $p$-adic integers, $p>3$. Siberian Mathematical Journal 54(3): 501-516.

Mukhamedov, F. \& Saburov, M. 2013. On equation $x^{q}=a$ over $\mathbb{Q}_{p}$. Journal of Number Theory 133(1): 55-58.

Neukirch, J. 1999. Algebraic Number Theory. Berlin, Heidelberg: Springer-Verlag.

Saburov, M. \& Ahmad, M.A. Kh. 2015. Solvability of cubic equations over $\mathbb{Q}_{3}$. Sains Malaysiana 44(4): 635-641.

Saburov, M. \& Ahmad, M.A. Kh. 2014. Solvability criteria for cubic equations over $\mathbb{Z}_{2}^{*}$ AIP Conference Proceedings 1602: 792-797.

Schikhof, W.H. 1984. Ultrametric Calculus: An Introduction to $p$-adic Analysis. Cambridge: Cambridge University Press.

Serre, J.P. 2003. On a theorem of Jordan. Bulletin of AMS. 40: 429-440.

Serre, J.P. 1979. Local Field. New York: Springer-Verlag.

Sun, Z.H. 2007. Cubic residues and binary quadratic forms. $J$. Numb. Theory 124(1): 62-104.

Sun, Z.H. 1998. On the theory of cubic residues and non-residues. Acta Arith. 4: 291-335.

Volovich, I.V. 1987. p-adic strings. Class. Quantum Gray. 4(4): 83-87.

Department of Computational \& Theoretical Sciences Faculty of Sciences, International Islamic University Malaysia P.O. Box 25200 Kuantan, Pahang Darul Makmur Malaysia

*Corresponding author; email: msaburov@gmail.com

Received: 26 April 2014

Accepted: 18 November 2014 